\title{
Air-Mobile Stroke Unit for access to stroke treatment in rural regions
}

\begin{tabular}{|r|l|}
\hline Journal: & International Journal of Stroke \\
\hline Manuscript ID & Draft \\
\hline Danuscript Type: & Review \\
\hline Complete List of Authors: & n/a \\
\hline & $\begin{array}{l}\text { Walter, Silke; Universitat des Saarlandes, Neurology } \\
\text { Zhao, Henry; Royal Melbourne Hospital, Faculty of Medicine, Dentistry and } \\
\text { Health Sciences, Neurology } \\
\text { Easton, Damien; Royal Melbourne Hospital, Faculty of Medicine, Dentistry } \\
\text { and Health Sciences, Neurology } \\
\text { Bil, Cees; RMIT University, Department of Aerospace Engineering } \\
\text { Sauer, Jonas; RMIT University, Department of Aerospace Engineering; FH } \\
\text { Aachen, Aerospace Engineering } \\
\text { Liu, Yang; Universitat des Saarlandes, Neurology } \\
\text { Lesmeister, Martin } \\
\text { Grunwald, Iris; Anglia Ruskin University, Chelmsford, Southend University } \\
\text { Hospital, Neuroscience Unit, Radiology } \\
\text { Donnan, Geoffrey; University of Melbourne, Florey Institute of } \\
\text { Neuroscience and Mental Health } \\
\text { Davis, Stephen; The Royal Melbourne Hospital, Department of Neurology } \\
\text { Fassbender, Klaus }\end{array}$ \\
\hline Keywords: & $\begin{array}{l}\text { Stroke, Thrombolysis, intraarterial treatment, mobile stroke unit, rural, } \\
\text { triage, flying doctor }\end{array}$ \\
\hline
\end{tabular}




\title{
Air-Mobile Stroke Unit for access to stroke treatment in rural regions
}

Silke Walter ${ }^{1,6^{*}}$, Henry Zhao ${ }^{2 *}$, Damien Easton ${ }^{2}$, Cees Bil ${ }^{3}$, Jonas Sauer ${ }^{3,4}$, Yang Liu ${ }^{1}$, Martin Lesmeister $^{1}$, Iris Q Grunwald ${ }^{5}$, Geoffrey A Donnan ${ }^{6}$, Stephen M Davis ${ }^{2}$, Klaus Fassbender $^{1}$

* Both authors contributed equally.

${ }^{1}$ Department of Neurology, Saarland University Medical Center, Kirrberger Strasse 100, 66421 Homburg, Germany

${ }^{2}$ Melbourne Brain Centre at the Royal Melbourne Hospital, Faculty of Medicine, Dentistry and Health Sciences, The University of Melbourne, Melbourne, Australia

${ }^{3}$ Department of Aerospace Engineering, RMIT University, GPO Box 2476, Melbourne 3001, Victoria, Australia

${ }^{4}$ Faculty of Aerospace Engineering, FH Aachen, University of Applied Sciences, Hohenstaufenallee 6, 52064 Aachen, Germany

${ }^{5}$ Neurocience Unit, Faculty of Medicine, Anglia Ruskin University, Chelmsford, Essex, UK; Radiology Department, Southend University Hospital NHS Trust, Essex, UK

${ }^{6}$ The Florey Institute of Neuroscience and Mental Health, The University of Melbourne, Melbourne, Australia

Correspondence to: Klaus Fassbender, Department of Neurology, Saarland University Medical Center, 66421 Homburg, Germany. Email: Klaus.Fassbender@uks.eu

\begin{abstract}
Abbreviations: CSC, comprehensive stroke centre; CT, computed tomography; EMS, emergency medical services; IAT, intraarterial therapy; IV, intravenous; LVO, large-vessel occlusion; MSU, Mobile Stroke Unit; POC, point of care; PSC, primary stroke centre; rt-PA,
\end{abstract}


recombinant tissue plasminogen activator

Key Words: stroke, thrombolysis, intraarterial treatment, rural, Mobile Stroke Unit, flying doctor, triage

Word Count: Total, 4072; abstract, 226; text, 2405

List of Tables and Figures: three figures 


\title{
Abstract
}

\section{Background:}

In recent years, important progress has been made in effective stroke treatment, however, patients living in rural and remote areas have nil or very limited access to timely reperfusion therapies.

\begin{abstract}
Aims:
Novel systems of care to overcome the detrimental treatment gap for stroke patients living in rural and remote regions need to be developed.
\end{abstract}

\section{Summary of review:}

A possible solution to the treatment disparity between stroke patients living in metropolitan and rural areas may involve use of specially designed aircrafts equipped with the ability to diagnose and treat acute stroke at remote emergency sites. We describe technical solutions for an Air-Mobile Stroke Unit (Air-MSU) concept, where an aircraft is customised with ability to perform multimodal computed tomography (CT), in addition to on-board laboratory equipment and telemedicine connection. The Air-MSU is envisioned not only to allow intravenous thrombolysis in the field but also to allow pre-hospital triage to a comprehensive stroke centre through use of contrast intracerebral vascular imaging. Several options for the Air-MSU concept are described, and issues regarding potential medical benefit, optimal operating environment, technical realization, integration in pre-existing solutions (e.g. flying doctor service), and cost-efficiency are addressed.

\section{Conclusion:}

The Air-MSU concept may represent a novel tool to reduce treatment disparity for stroke patients in rural and remote areas. However, this approach requires further implementation research to determine the overall benefit to these communities. 


\section{Background}

Stroke is the second highest cause of death, disability, and dementia worldwide, ${ }^{1-3}$ and prevalence is expected to increase due to the rising aged population in many countries. ${ }^{4}$ Apart from considerable suffering for patients and their families, stroke also results in enormous costs for society, including those associated with loss of employment and long-term care expenses. 5,6

Although acute ischaemic stroke is a treatable disease, timely access to guidelineadherent acute reperfusion therapies is a major limitation for many areas of the world, especially in countries with large rural and remote areas, where the coverage of specialized stroke centres is very poor.

\section{Treatment disparity for stroke patients living in rural regions}

Within a single country, the rural setting and distances to the nearest stroke centre are key factors in the delivery of time-sensitive stroke treatment. ${ }^{7,8}$ Reported times from symptom onset to admission to rural hospitals range from 5 to 30 hours. ${ }^{9,10}$ These pre-hospital delays have subsequently been attributed as a major reason for the very low thrombolysis rates of 1$6 \%$ for patients in rural areas worldwide. ${ }^{11-13}$ In Australia, only $3 \%$ of patients in rural areas were able to access a stroke unit in a timely manner, compared to $77 \%$ of patients in metropolitan areas. ${ }^{14}$ Beside, patients living in rural areas are less likely to receive brain imaging within 24 hours, carotid imaging, and consultations from neurologists. ${ }^{15}$ This affects not only low-income countries, but also those with middle- and high-incomes. ${ }^{9}$ The US Get With The Guidelines-Stroke registry has identified arrival at a rural hospital as one factor associated with recombinant tissue plasminogen activator (rt-PA) treatment failure. ${ }^{16}$ Even worse, the rural-urban disparity in rt-PA administration has increased in the last decades. ${ }^{17}$ The treatment gap between metropolitan and rural is even more pronounced with 
endovascular treatment options for acute stroke. ${ }^{18}$ This is due to the requirement for substantial and highly specialized training, technical resources and concentrated periinterventional services (neurosurgery, anaesthesiology, and neurointensive care) to allow effective delivery of intraarterial therapy (IAT) services. This complex infrastructure is available only at a few specialized stroke centres (comprehensive stroke centres, CSCs), which are almost exclusively located in metropolitan centres. ${ }^{19}$ Patients living in rural and remote regions, therefore, have only limited access to IAT services in time. ${ }^{20}$ However, it must be considered that for acute stroke patients from remote areas $(\leq 10,000$ inhabitants, Rural Remote and Metropolitan Areas classification, RRMAS ${ }^{21}$ ) it is not all about getting a faster treatment, but about getting any treatment, which could represent their only chance of recovery considering the latest DAWN recanalization trial results. ${ }^{22}$

\section{Mobile Stroke Unit (MSU) concept}

The MSU concept is a recent development, which allows diagnosis and treatment of stroke patients in the pre-hospital setting. This is based on a specialised ambulance that is additionally equipped to allow pre-hospital imaging and triage, including a computed tomography (CT) scanner for multimodal imaging studies, point-of-care (POC) laboratory for blood tests required for treatment, ${ }^{23,24}$ and telemedicine connection to a stroke specialist.

Several studies have shown that use of an MSU significantly reduces time delays prior to thrombolysis. ${ }^{25-28}$ Additionally, the proportion of patients that are able to be treated within 60 min after stroke symptom onset ("the golden hour of stroke") have risen dramatically, from $4 \%$ to $57 \%{ }^{25}$ This is due not only to a reduction in transport times, but also to the marked improvement in efficiency from involvement of a specialised multi-disciplinary stroke team that operates alongside the MSU. ${ }^{29}$

Another important benefit of MSU is the use of pre-hospital CT to allow triage of 
patients with large-vessel occlusion (LVO) or haemorrhage to the closest centre with IAT or neurosurgical capability. This reduces the time to treatment for these important interventions and avoids costly inter-hospital transfers.

\section{Air-Mobile Stroke Unit (Air-MSU) for delivery of acute stroke treatment to rural patients}

A potential solution that could reduce the detrimental treatment disparity between stroke patients living in urban areas and those living in rural or remote areas could be the use of the MSU concept of "bringing the stroke treatment to the patient". In line e.g., with the already established Royal Flying Doctor Service of Australia strategy (https://www.flyingdoctor.org.au), an airplane (Figure 1) or helicopter (Figure 2) Air-MSU could be equipped identically to an MSU ambulance, with multimodal imaging capabilities, a POC laboratory, and a telemedicine connection to the CSC so that stroke patients in remote regions could obtain diagnosis and treatment close at the emergency site.

\section{Technical solutions}

Transferring the MSU concept to an aircraft may be realized e.g., by using a fixed wing aircraft such as the Hawker 800 XP (BAE Systems, London, UK) or the Pilatus PC-24 (Pilatus Aircraft Ltd, Stans, Switzerland), that are already in use by the Royal Flying Doctor Service of Australia. Such Air-MSUs would have to carry, in addition to all of the usual medical equipment of a flying ambulance, a) an accumulator-driven CT scanner allowing multimodal imaging (e.g., CereTom; Samsung Neurologica Corporation, Danvers, MA, USA), b) a telemedicine system (e.g. MEYTEC GmbH, Werneuchen, Germany) enabling the transmission of digital imaging and communication data obtained by CT scanning or video of clinical examination to the hospital's picture archiving and communication system (PACS) 
and c) a POC laboratory system for blood tests depending on local guidelines: blood count (e.g., pocH-100i; Sysmex Europe GmbH, Hamburg, Germany); international normalised ratio and activated partial thromboplastin time (e.g., Hemochron Jr. Signature; International Technidyne Corporation, Edison, NY, USA); and liver enzymes, creatinine level, and glucose concentration (e.g., Reflotron Plus V Clinical Chemistry Analyzer; Roche Diagnostics, Mannheim, Germany).

In addition, the Air-MSU would carry a lightweight patient stretcher with the ability to bring the patient into alignment for a CT scan, and at least three seats for the medical staff. Figure 1 shows a model of the Pilatus PC24 cabin that was built up for the purpose of visualizing how the equipment could be positioned and how much working space remains for the medical staff. The geometry of the CT scanner allows only a positioning in the middle of the cabin's longitudinal axis, which means that the stretcher also needs to be positioned accordingly. This is rather unusual for fixed wing air ambulance aircraft. However, the cabin of the PC24 offers enough space for medical staff to work around the patient from both sides.

In aviation the weight-distribution and -limitation is crucial as it directly influences the flight performance of the respective aircraft. As the CT scanner represents a relatively heavy single item for a typical air ambulance aircraft, it should be placed as near to the centre of gravity as possible. But the CT scanner will also block a big part of the cabin's cross section, so to guarantee a smooth workflow only positioning in the front or the rear of the cabin seems reasonable. From these two options, the one closer to the centre of gravity should be chosen. However, this option might not be available in case access to the cabin is only possible through one door in the front or rear of the cabin, leaving the respective other side as the only option to place the CT scanner (Figure 1). Nevertheless, it should be kept in mind that other equipment might have to be positioned in a way that the weight of the $\mathrm{CT}$ scanner is counterbalanced. In any event, because of the aforementioned influence on performance, it 
would be preferable to design the CT scanner as light and small as possible for an Air-MSU. One idea to achieve this even with e.g., the Ceretom CT scanner is to use the Auxiliary Power Unit (APU), as power supply instead of the integrated batteries.

For a reliable and durable operation of the Air-MSU, mainly the CT scanner, but also the rest of the necessary equipment has to show resistance to vibration, humidity and sudden loss of pressure, and exclude electromagnetic interference with the avionics of the aircraft. This means that a test period will be needed and depending on the results the development of additional systems like for example a damping system may be required.

A similar fixation with bolts like in the ground-bound MSU could be used to mount the CT scanner to the cabin floor. But it will need to be designed for higher loads because regulations demand that equipment withstands high G-forces in case of an emergency landing. In addition, these loads make it necessary that the cabin floor will have to be reinforced where the CT scanner is fixed. Furthermore, to get the CT scanner into a small fixed-wing aircraft like the PC24, it will be necessary to dismantle the CT scanner and assemble it again in the cabin. In total, we expect an additional weight to the aircraft of approximately $400 \mathrm{~kg}$, a feasible amount considering the maximum payload of air ambulance aircraft, which provide a cabin that is big enough to accommodate the scanner. Nevertheless, the impact of the additional payload on the available range needs to be considered when it comes to the question how big the area should be that is to be covered by the Air-MSU.

Alternatively, the same concept could also be realized by using a helicopter, although the space and weight restrictions may be more challenging than those of a fixed-wing aircraft. A case study has shown that even the cabins of conventional emergency medical services (EMS) helicopters like the Augusta Westland 139 (Leonardo S.p.A., Rome, Italy) or the H155 (Airbus Helicopters, Marignane, France) do not provide enough height to accommodate the Ceretom CT scanner. Furthermore, even if only equipped with what is absolutely necessary 
for an EMS mission, additional 400kg would lead to a surpassing of their maximum take-off weight. As a result, a larger multimission helicopter like the Augusta Westland 101 (Leonardo S.p.A., Rome, Italy) or the NH90 (NHIndustries, Aix-en-Provence, France) will be needed. Those helicopters provide not only a sufficiently large cabin to accommodate the Ceretom CT scanner but also offer high maximum payload, making an integration straight forward. A similar model like the one of the PC24 but for the NH90 can be seen in Figure 2. Additional advantage of such a helicopter is that it is much easier to load the patients into the cabin and bring them into correct alignment with the $\mathrm{CT}$ scanner, as they usually have a ramp in the rear of the cabin. This results in positioning the CT scanner in the front of the cabin as the only choice.

However, as they are not routinely used for EMS purposes, this concept would come with the additional investment costs of such a helicopter, and its higher weight would limit the options for landing on a hospital helipad. The damping of the CT scanner should also be done more carefully, as helicopters are usually a much bigger source of vibration than fixed-wing aircrafts. Obvious advantage of using a helicopter instead of a fixed-wing aircraft is that no runway is needed but landing directly at the emergency site or hospital would be possible. Moreover, the cabin is usually wider, providing more space for medical staff to work around the patient. The fixed-wing aircraft however remains superior in range and speed, and might therefore reach some remote areas a helicopter cannot.

\section{Different staffing options of an Air-MSU}

Regarding the staffing, different options exist that depend on the size and level of competence of the regular flying doctor team. As a starting point under still experimental conditions staff consisting of a paramedic, a radiographer and a general practitioner trained in acute stroke 
medicine or even a neurologist with emergency medical training might be beneficial based on experiences in ground-bound MSUs. ${ }^{30,31}$ In addition, telemedical consultation including transfer of CT images of the Air-MSU to the next CSC supports the emergency team in diagnosis, treatment decision and initiation.

\section{Different scenarios of Air-MSU missions}

Two main scenarios can be anticipated in use of an Air-MSU in order to improve access to state-of-the art stroke treatment in remote areas (Figure 3):

In scenario 1, the Air-MSU, having been activated by the EMS dispatch centre, reaches the patient at the emergency site, using the closest possible landing option. The AirMSU thereby may either function as first responding EMS or takes over the stroke patient from the first responding regional EMS. There, all stroke examinations (neurological examination, imaging, and POC laboratory testing) are performed already in the pre-hospital phase of stroke management, and, if indicated, IV thrombolysis is initiated in the field. Moreover, based on the clarification of the vascular cause directly at the emergency site, a correct triage decision about the appropriate target hospital (primary stroke centre (PSC) versus CSC) can be made. In case of LVO or intracerebral haemorrhage, the Air-MSU may, after pre-notification, transfer the patient directly to a CSC for required endovascular, neurosurgical, or neurointensive treatment. All other cases may be triaged to the nearest regional hospital (primary hospital or PSC).

In scenario 2, the patient has already been admitted to a primary hospital or PSC without acute stroke treatment, e.g. thrombectomy capability. The Air-MSU team, alerted either by the EMS dispatch office or by the hospital team, would come to take over the specialized stroke management. This includes, apart from an eventually indicated IV thrombolysis, a vascular diagnostic work up by CT-angiography and, eventually, CT 
perfusion, as precondition for a rational triage decision about whether or not the patient should be secondarily transferred to a CSC for IAT. Alternatively, in the rural hospital the patient could, while the Air-MSU is still underway, obtain first neurological assessment and, after non-contrast imaging, thrombolytic treatment. After arrival, the Air-MSU would complement stroke management with vascular imaging as a precondition for a rational triage decision, and, if indicated, realizes the transport of the patient to the CSC for specialized stroke treatment.

\section{Conclusion}

Novel health care solutions are urgently needed that reduce the detrimental treatment gap for stroke patients living in rural and remote regions. This is especially true with regard to the poor access to timely reperfusion therapies outside of major metropolitan areas. The Air-MSU concept allows pre-hospital assessment, imaging, blood analysis and specialized treatment by incorporating a portable CT scanner platform, laboratory unit and telemedicine connection together with a stroke specialised team aboard an aircraft. This would allow overall access and dramatically reduced time delays for reperfusion therapies and enable correct pre-hospital triage to endovascular and neurosurgical centres, thereby reducing the treatment disparity for geographically isolated patients. Further research is needed to establish optimal technical solutions and geographical implementation as well as cost-effectiveness of such an approach. 


\section{Figure Legends}

Figure 1. Design of an Air-Mobile Stroke Unit (Air-MSU), including a computed tomography (CT) scanner, a point-of-care (POC) laboratory and telemedicine equipment, a lightweight stretcher, and four additional seats for medical staff. As exemplary basis of such system, a Pilatus PC24, which is used by the Royal Flying Doctor Service of Australia is shown.

Figure 2. Design of an Air-Mobile Stroke Unit (Air-MSU), integrated in the cabin of the NH90 multimission helicopter.

Figure 3. Two scenarios of Air-Mobile Stroke Unit (Air-MSU) missions. Scenario 1: The airplane lands at a field close to the emergency site. At this location the Air-MSU team either functions as first responding emergency medical services (EMS) unit or meets the regional EMS to take over specialized neurological assessment including imaging studies and laboratory tests. The patient may receive pre-hospital thrombolysis and based on the results of vascular imaging (CT-angiography, CT-perfusion) a decision between treatment in a comprehensive stroke centre (CSC) versus a primary hospital or primary stroke centre (PSC) will be made. Scenario 2: The patient is first admitted to a regional primary hospital by the regular EMS. The Air-MSU, then, accesses the patient at this hospital and takes over specific stroke work up including vascular imaging. Depending on the results of these advanced diagnostic procedures the patient may, after eventually indicated IV thrombolysis, be either transported to a CSC for specialized acute stroke treatment or remains at the first admitting rural hospital. 


\section{Funding: None}

\section{Conflict of interest: None}

\section{Author contribution:}

Silke Walter: conception, design, drafting of the manuscript and final approval

Henry Zhao: design, drafting and final approval of the manuscript

Damien Easton: conception, design, final approval of the manuscript

Cees Bil: conception, design, drafting of the manuscript, final approval of the manuscript

Jonas Sauer: conception, design, final approval of the manuscript

Yang Liu: critical discussion and final approval of the manuscript

Martin Lesmeister: critical discussion and final approval of the manuscript

Iris Q Grunwald: critical discussion and final approval of the manuscript

Geoffrey A Donnan: conception, design, drafting of the manuscript and final approval

Stephen M Davis: conception, design, drafting of the manuscript and final approval

Klaus Fassbender: conception, design, drafting of the manuscript and final approval 


\section{References}

1. WHO. The top 10 causes of death, fact sheet, updated January 2017 , http://www.who.int/mediacentre/factsheets/fs310/en/ (accessed 8 November 2017).

2. Ovbiagele B, Goldstein LB, Higashida RT, et al. American Heart Association Advocacy Coordinating Committee and Stroke Council. Forecasting the future of stroke in the United States: a policy statement from the American Heart Association and American Stroke Association. Stroke 2013; 44: 2361-2375.

3. Mozaffarian D, Benjamin EJ, Go AS, et al. Heart disease and stroke statistics-2016 update: a report from the American Heart Association. Circulation 2016; 133: e38-e360.

4. Feigin VL, Norrving B and Mensah GA. Global burden of stroke. Circ Res 2017; 120: $439-448$.

5. Olesen J, Gustavsson A, Svensson M, et al. The economic cost of brain disorders in Europe. Eur J Neurol 2012; 19: 155-162.

6. Roger VL, Go AS, Lloyd-Jones DM, et al. Heart disease and stroke statistics--2012 update: a report from the American Heart Association. Circulation 2012; 125: e2-e220.

7. Cox AM, McKevitt C, Rudd AG and Wolfe CD. Socioeconomic status and stroke. Lancet Neurol 2006; 5: 181-188.

8. Teuschl Y and Brainin M. Stroke education: discrepancies among factors influencing prehospital delay and stroke knowledge. Int J Stroke 2010; 5: 187-208.

9. Joubert J, Prentice LF, Moulin T, et al. Stroke in rural areas and small communities. Stroke 2008; 39: 1920-1928.

10. Oliveira-Filho J, Martins SC, Pontes-Neto OM, et al. Guidelines for acute ischaemic stroke treatment: part I. Arq Neuropsiquiatr 2012; 70: 621-629.

11. Cadilhac DA, Purvis T, Kilkenny MF, et al. Evaluation of rural stroke services: does implementation of coordinators and pathways improve care in rural hospitals? Stroke 
2013; 44: 2848-2853.

12. Ganesh A, Camden M, Lindsay P, et al. The quality of treatment of hyperacute ischaemic stroke in Canada: a retrospective chart audit. CMAJ Open 2014; 2: E233-E239.

13. Newbury J, Kleinig T, Leyden J, et al. Stroke Epidemiology in an Australian Rural Cohort (SEARCH). Int J Stroke 2017; 12: 161-168.

14. Eissa A, Krass I and Bajorek BV. Optimizing the management of acute ischaemic stroke: a review of the utilization of intravenous recombinant tissue plasminogen activator (tPA). J Clin Pharm Ther 2012; 37: 620-629.

15. Koifman J, Hall R, Li S, et al. The association between rural residence and stroke care and outcomes. J Neurol Sci 2016; 363: 16-20.

16. Messé SR, Khatri P, Reeves MJ, et al. Why are acute ischemic stroke patients not receiving IV tPA? Results from a national registry. Neurology 2016; 87: 1565-1574.

17. Gonzales S, Mullen MT, Skolarus L, Thibault DP, Udoeyo U and Willis AW. Progressive rural-urban disparity in acute stroke care. Neurology 2017; 88: 441-448.

18. Prabhakaran S, Ruff I and Bernstein RA. Acute stroke intervention: a systematic review. JAMA 2015; 313: 1451-1462.

19. Menon BK, Campbell BC, Levi C and Goyal M. Role of imaging in current acute ischaemic stroke workflow for endovascular therapy. Stroke 2015; 46: 1453-1461.

20. Suzuki S, Saver JL, Scott P, et al. Access to intra-arterial therapies for acute ischaemic stroke: an analysis of the US population. AJNR Am J Neuroradiol 2004; 25: 1802-1806.

21. Australian Institute of Health and Welfare 2004. Rural, regional and remote health: a guide to remoteness classifications. AIHW cat. no. PHE 53. Canberra: AIHW.

22. Nogueira RG, Jadhav AP, Haussen DC, et al. Thrombectomy 6 to 24 Hours after Stroke with a Mismatch between Deficit and Infarct. N Engl J Med 2018; 378: 11-21.

23. Boehringer Ingelheim Limited. Actilyse. Summary of Product Characteristics, 2017. 
www.medicines.org.uk/emc/medicine/308 (accessed 26 September 2017).

24. Jauch EC, Saver JL, Adams HP Jr, et al. Guidelines for the early management of patients with acute ischaemic stroke: a guideline for healthcare professionals from the American Heart Association/American Stroke Association. Stroke 2013; 44: 870-947.

25. Walter S, Kostopoulos P, Haass A, et al. Diagnosis and treatment of patients with stroke in a mobile stroke unit versus in hospital: a randomised controlled trial. Lancet Neurol 2012; 11: 397-404.

26. Ebinger M, Winter B, Wendt M, et al. Effect of the use of ambulance-based thrombolysis on time to thrombolysis in acute ischaemic stroke: a randomized clinical trial. JAMA 2014; 311: 1622-1631.

27. Bowry R, Parker S, Rajan SS, et al. Benefits of stroke treatment using a Mobile Stroke Unit compared with standard management: the BEST-MSU Study Run-In Phase. Stroke 2015; 46: 3370-3374.

28. Itrat A, Taqui A, Cerejo R, et al. Telemedicine in prehospital stroke evaluation and thrombolysis: taking stroke treatment to the doorstep. JAMA Neurol 2016; 73: 162-168.

29. Fassbender K, Grotta JC, Walter S, Grunwald IQ, Ragoschke-Schumm A and Saver JL. Mobile stroke units for prehospital thrombolysis, triage, and beyond: benefits and challenges. Lancet Neurol 2017; 16: 227-237.

30. Walter S, Kostopoulos P, Haass A, et al. Bringing the hospital to the patient: first treatment of stroke patients at the emergency site. PLoS One 2010; 5: e13758.

31. Ragoschke-Schumm A, Walter S, Haass A, et al. Translation of the 'time is brain' concept into clinical practice: focus on prehospital stroke management. Int J Stroke 2014; 9: 333340. 


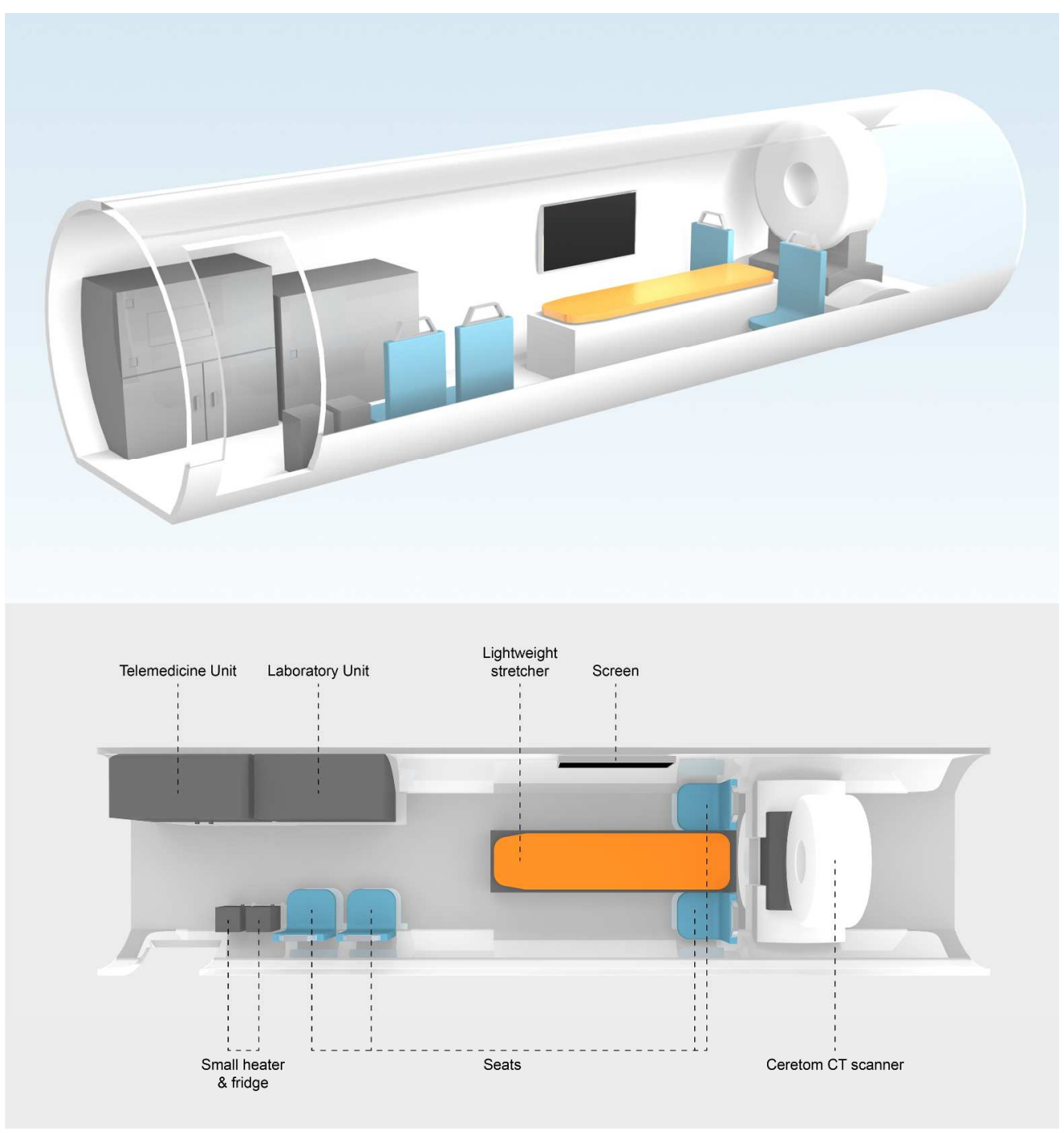

Design of an Air-Mobile Stroke Unit (Air-MSU), including a computed tomography (CT) scanner, a point-ofcare (POC) laboratory and telemedicine equipment, a lightweight stretcher, and four additional seats for medical staff. As exemplary basis of such system, a Pilatus PC24, which is used by the Royal Flying Doctor Service of Australia is shown.

$400 \times 422 \mathrm{~mm}(300 \times 300 \mathrm{DPI})$ 


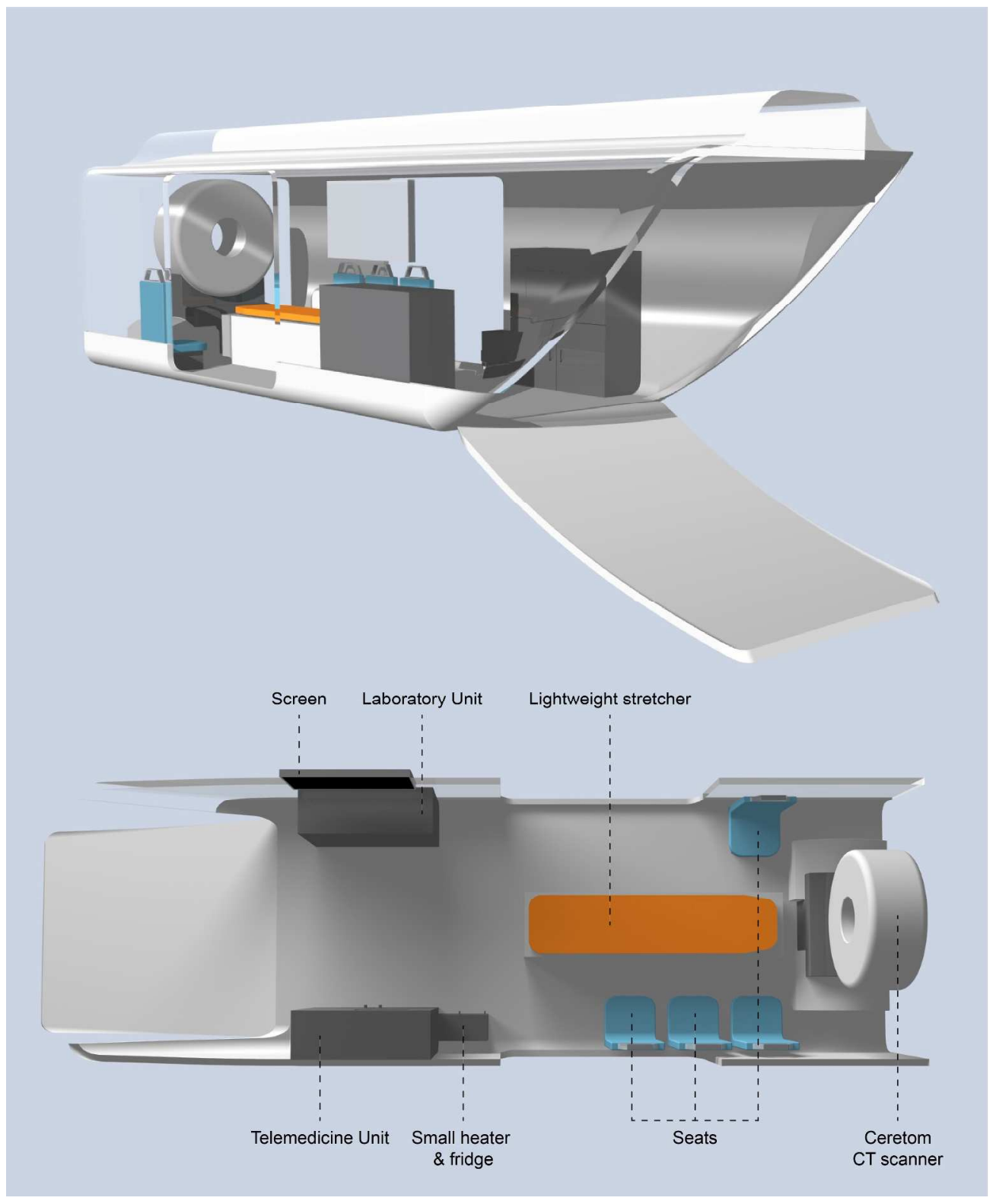

Design of an Air-Mobile Stroke Unit (Air-MSU), integrated in the cabin of the NH90 multimission helicopter. $315 \times 381 \mathrm{~mm}(300 \times 300$ DPI $)$ 


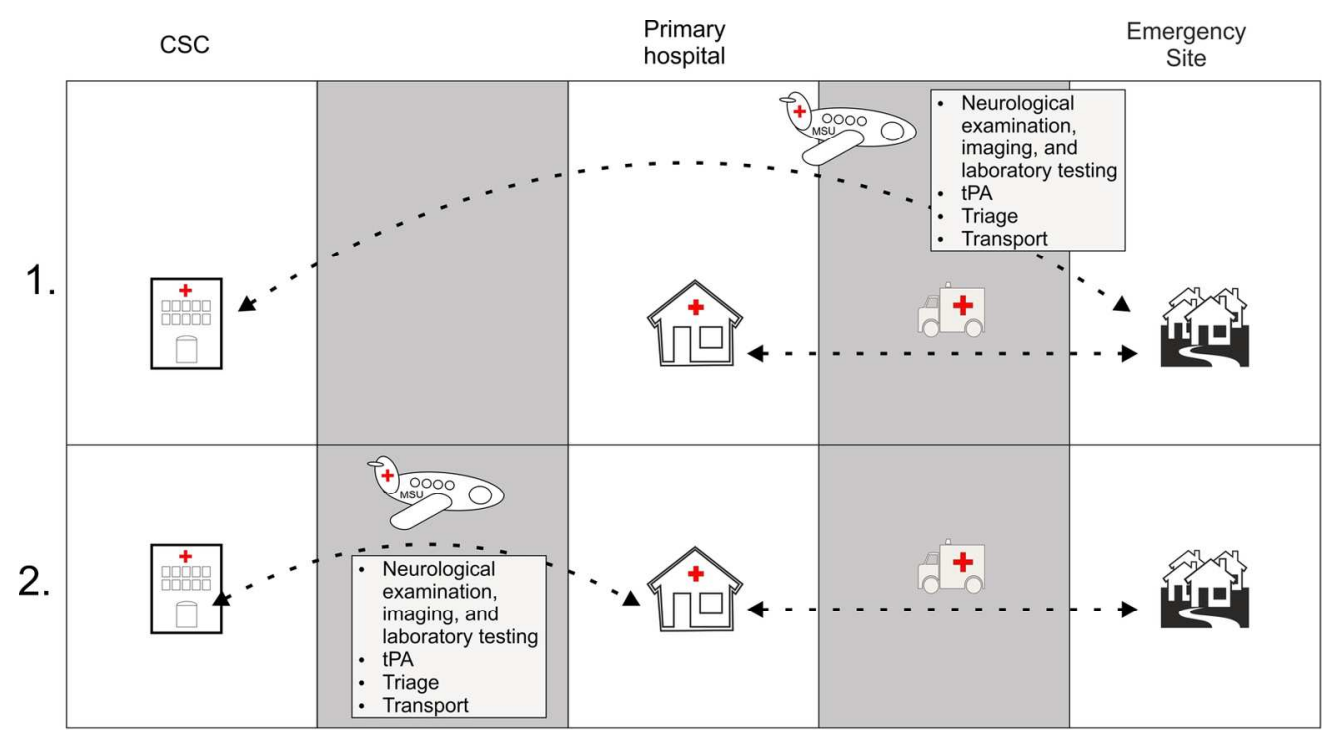

Two scenarios of Air-Mobile Stroke Unit (Air-MSU) missions. Scenario 1: The airplane lands at a field close to the emergency site. At this location the Air-MSU team either functions as first responding emergency medical services (EMS) unit or meets the regional EMS to take over specialized neurological assessment including imaging studies and laboratory tests. The patient may receive pre-hospital thrombolysis and based on the results of vascular imaging (CT-angiography, CT-perfusion) a decision between treatment in a comprehensive stroke centre (CSC) versus a primary hospital or primary stroke centre (PSC) will be made. Scenario 2: The patient is first admitted to a regional primary hospital by the regular EMS. The Air-MSU, then, accesses the patient at this hospital and takes over specific stroke work up including vascular imaging. Depending on the results of these advanced diagnostic procedures the patient may, after eventually indicated IV thrombolysis, be either transported to a CSC for specialized acute stroke treatment or remains at the first admitting rural hospital.

$147 \times 80 \mathrm{~mm}(300 \times 300$ DPI $)$ 